\title{
ALICE Commissioning
}

\author{
Sylvain Chapeland ${ }^{12}$ \\ CERN \\ Geneva, Switzerland \\ E-mail: sylvain.chapeland@cern.ch
}

\begin{abstract}
ALICE (A Large Ion Collider Experiment) is the heavy-ion detector designed to study the physics of strongly interacting matter and the quark-gluon plasma at the CERN Large Hadron Collider (LHC). This paper outlines the commissioning of the ALICE detector. We briefly describe the experiment layout, and then review the commissioning activities that took place in order to prepare the experiment for the first beam. We present the goals and achievements of the various phases, including several global cosmic runs, and comment on the key issues that had to be dealt with during this critical but successful start-up phase.
\end{abstract}

2008 Physics at LHC

Split, Croatia

September 29 - 4 October 2008

Speaker

for the ALICE collaboration 


\section{Introduction}

ALICE (A Large Ion Collider Experiment) [1][2][3][4], is the heavy-ion detector designed to study the physics of strongly interacting matter and the quark-gluon plasma at the CERN Large Hadron Collider (LHC).

\subsection{Experiment layout}

ALICE currently consists of 16 sub-detectors, being able to take data independently (standalone operation) or in global partitions (set of sub-detectors running together). All 16 subdetectors can run standalone in parallel, and up to 6 global partitions can run in parallel. The detector includes high resolution tracking (silicon detectors, large time-projection chamber), particle identification, and triggering elements. It features two large magnets, a main solenoid and a dipole on the Muon arm. More information on the detector layout can be found in another paper of this conference [5].

In addition to the sub-detectors, five online systems [6] handle the facilities and data flow:

- The Experiment Control System (ECS) controls and synchronizes all the online systems, and provides a single entry point for the operation of the whole experiment.

- The Data Acquisition system (DAQ) handles the data flow from the detector to the permanent data storage in the CERN computing center.

- The Detector Control Systems (DCS) controls the sub-detectors and their facilities.

- The Central Trigger Processor (CTP) provides the trigger signals to readout the subdetectors based on the trigger sub-detectors inputs.

- The High-Level Trigger (HLT) provides a software filtering mechanism to select interesting events in order to optimize use of the recording bandwidth available.

The experiment also includes the offline analysis facilities, and online monitoring and promptreconstruction provide feedback on data quality at run time.

All these components had to be commissioned, first standalone, and then interacting with the other entities of the experiment.

\subsection{Individual sub-detector commissioning sequence}

Each sub-detector was commissioned individually before being joined to a global partition. This sequence started by the hardware and firmware installation, checking and tuning. The first step to integrate the sub-detector in the global system consisted in the validation of its interfaces to the online systems. Once done, tests of standalone data taking and stability exercise were performed, including taking data with pulser trigger up to $40 \mathrm{MHz}$, random triggers, and cosmics. Necessary fixes were applied and re-tested until a perfectly stable and smooth operation was achieved. At this point, all calibration and control operations were also verified and practiced. Finally, tests were done with each new sub-detector running in a global partition together with other sub-detectors. Given the usual iterations needed, the time for the whole procedure could take up to several weeks for some sub-detectors. 


\section{ALICE commissioning timeline}

The ALICE commissioning took place between December 2007 and September 2008. There were three global runs:

- $1^{\text {st }}$ global run from $10^{\text {th }}$ to $21^{\text {st }}$ December 2007

- $2^{\text {nd }}$ global run from $4^{\text {th }}$ February to $9^{\text {th }}$ March 2008

- $3^{\text {rd }}$ global run from $5^{\text {th }}$ May until $12^{\text {th }}$ October 2008

The data taking activity closely matched the global run periods, steadily ramping up through the year, both in data taking time and aggregated amount of data, as seen on Figure 1 (left and right respectively). In total, more than 3.5 PB of data were readout from the detector, and a total of $320 \mathrm{~TB}$ were recorded to tape
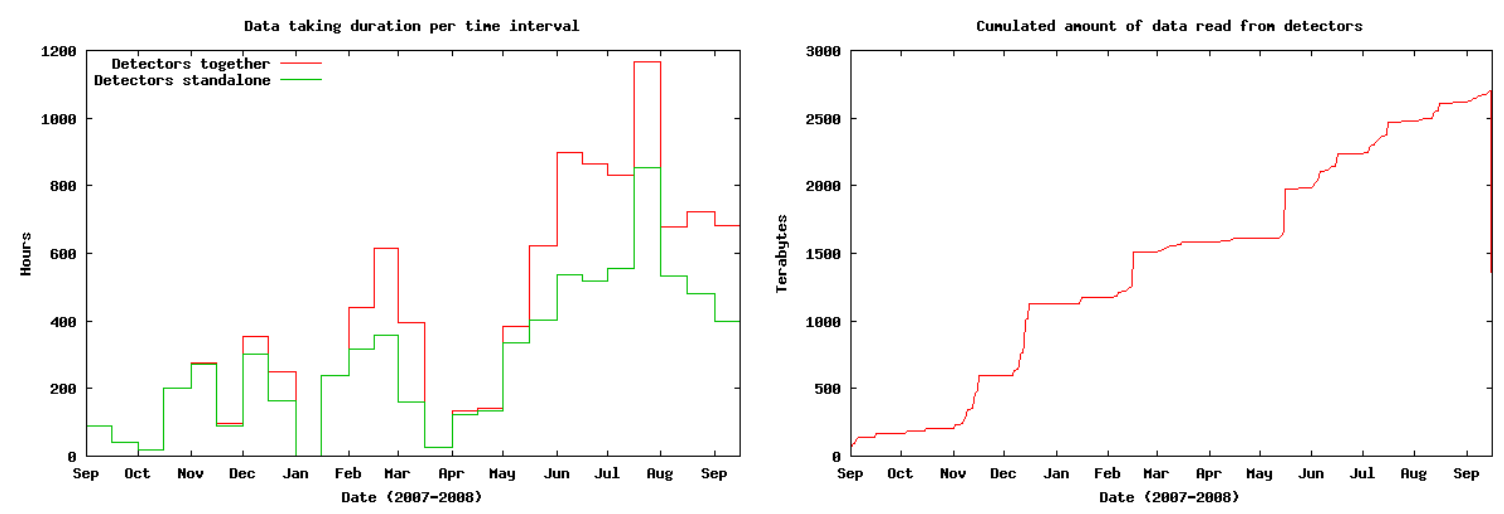

Figure 1 - data taking time and amount of data readout from ALICE detector

The hardware installation lasted until beginning of June 2008, and uninterrupted 24/7 experiment operation started in May until October 2008.

The first particles from the LHC machine were seen on 15th June by the Silicon Pixel detector (SPD) which recorded muon tracks produced in the beam dump near the experimental area (“Point 2"), as seen on Figure 2.

Further machine interaction occurred on 8th August and 24th August during injection tests, where precious timing data were collected to align trigger input signals.

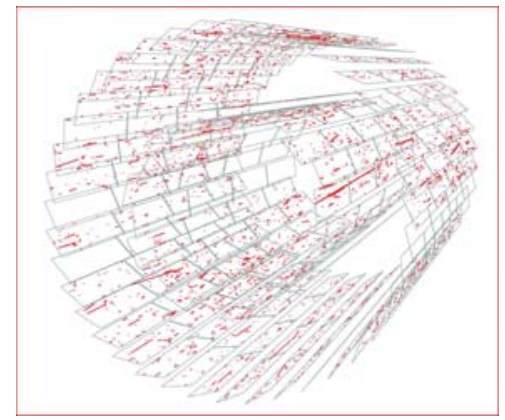

Figure 2- First LHC particles seen by $S P D$ on $15 / 06 / 2008$

Very high fluxes were measured during the beam dumps, and even during injection through ALICE (10s to 1000 s particles $/ \mathrm{cm}^{2}$ ) with beam screens in LHC and/or TI2. It was therefore decided to switch off all sensitive detectors during injection. 


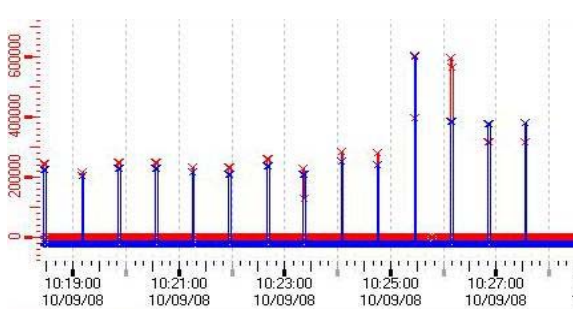

Figure 3 - V0 luminosity monitor with first beam looping $\mathrm{LHC}$
The first circulating beam occurred on 10th September 2008. The first beam data are only available for a limited set of detectors: SPD and V0 were always on (trigger), whereas Silicon Strip Detector (SSD), Silicon Drift Detector (SDD), Forward Multiplicity Detector (FMD), and T0 (Cherenkov counters) only occasionally. The evidence of first beam looping LHC was captured by the V0 luminosity monitor, as seen on Figure 3 showing the signal doubling when the beam starts to pass twice through ALICE.

During the short period with beam in LHC, some interesting events were even detected, as on Figure 4. It shows a collision that occurred inside the SPD on $11^{\text {th }}$ September, with the reconstructed tracks.

\subsection{Global runs}

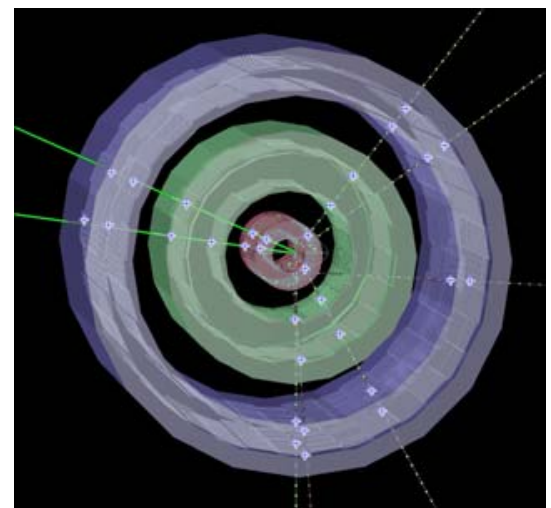

Figure 4- Collision inside silicon pixel detector

The global runs were an excellent way of exercising and understanding operation, performance and stability of the experiment. It was also a unique opportunity to collect data (cosmic events, lasers, beam injections) in order to perform precise alignment and calibration of the sub-detectors.

The main achievement of the first global run was to run detectors together and operate the system continuously during two weeks. The second global run was targeted at operating the magnets, running more detectors, and collect alignment data. The third global run aimed at performing further calibrations and alignment measurements, and at tuning the system for operation with the LHC beam.

\subsection{Calibration and alignment}

Some of the calibration tasks are performed directly online in dedicated runs (e.g. pedestals calculation), and 35 such tasks are operational. Heavier tasks are completed offline, such as (here are some examples for the Time Projection Chamber (TPC)): gain calibration with krypton data, drift velocity measurement with laser tracks, ExB scan with magnet operation.

Alignment of the detectors is achieved with cosmics data. The cosmic runs provided enough data for a good alignment of the SPD, with a residual misalignment of less than $10 \mu \mathrm{m}$ using 55k cosmic muon hits (@ 0.1Hz). SSD, SDD and ITS/TPC alignments have started but are not complete yet. 


\subsection{Achievements}

As regards control, the DCS ensures a safe and reliable operation of hardware. It provides device configuration, control and monitoring, with tasks like powering on and off equipment, front-end electronics configuration, switching to beam-tuning states, and handling interlocks. The system has 1600 servers publishing 150000 services, reflecting the state of $10^{8}$ registers, with 1200 network attached devices, and a sustained database archiving rate of 1000 updates per second.

The ECS provides the synchronization of online systems, including the execution of ordered tasks at start / end of run. Operations depend on run type, and more than 30 run types were exercised. The list and types of operation is still evolving and growing to match new requirements arising with operation.

The CTP was tested with realistic configurations, using simulated random and cosmic triggers, with parallel detectors clusters and parallel trigger classes. The timing of trigger input was tuned, as well as the synchronization with LHC signals.

Data taking with the HLT allows online reconstruction and data reduction. All operation modes were exercised, including with event-reject decisions applied. The HLT features reconstruction of the main detectors (TPC, Transition Radiation Detector (TRD), Photon Spectrometer (PHOS), Muon Arm), and online compression for SDD.

The DAQ proved to perform recording at high data rate, adequate for p-p collisions: up to 400 $\mathrm{MB} / \mathrm{s}$ sustained, and even $1.3 \mathrm{~GB} / \mathrm{s}$ for short periods (limited by transient storage space available at the experimental area). A wide range of operating conditions were tested, from the slow cosmic event rates to high recording speeds, with concurrent detector activity and flexible configurations. The usual run duration in global partitions (sub-detectors running together) is usually between 1 and 10 hours, as seen on Figure 5

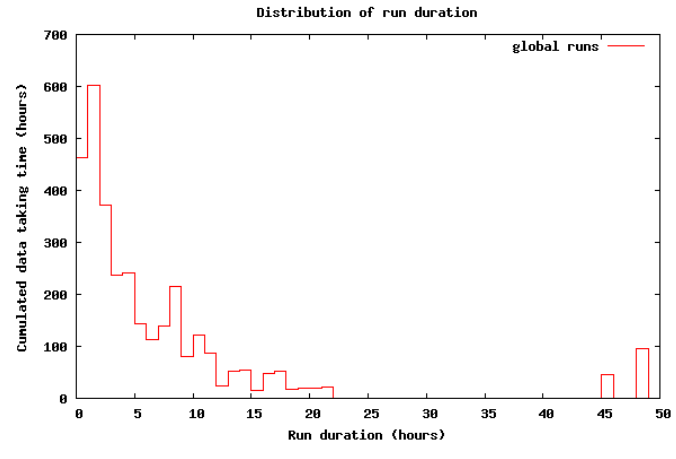

Figure 3- Distribution of run duration for global partitions

Concerning Offline, the GRID was used with success, including RAW data registration and replication, workload management, and storage. It used the full reconstruction chain for RAW and Event Summary Data (T0, T1s and T2s). More than 1 million user jobs were submitted by more than 130 active users, which averages around, 2.7K per day.

Dedicated clusters for Time-critical data analysis were used (CAF, GSIAF), and test of reconstruction algorithms on cosmics events, injections, and lasers were performed. The alignment, efficiencies, and calibration measurements were produced with success.

The experiment was operated 24/7 during the global runs, which accounts to more than 4300 shifts between December 2007 and August 2008. A strict remote access policy was used: all systems run on an isolated network, which ensured no security issues. The experiment 
logbook was heavily used, to log and report operations (both manually and automatically by adequate processes). It proves to be an excellent source of data to understand and check how things behaved during the global runs. The online monitoring and data quality monitoring tools developed and used during the commissioning were helpful to spot detectors issues rapidly and ensure that recorded data are worthy.

\subsection{Issues solved and findings}

The achievements were however not effortless, and here is a summary of some of the issues handled during commissioning:

- We had a few issues with 'simple' services such as cooling and power supplies

- Much work was needed on detectors firmware and noise issues

- The scalability of the control required some tuning, and we had to distribute and isolate some systems

- The CPU needed for data formatting is higher than anticipated (which is the price to pay for a faster offline reconstruction)

- The geographical mapping of data links required some iterations

- The test of all (complex!) trigger configurations was time consuming. A fix was needed for spurious triggers.

- The number of problems grows with the number of detectors involved, and starting a global run takes initially some time.

- The detector state machines and complex sequence control is still under development, and not yet fully automated

\section{Conclusion}

The commissioning of the ALICE experiment was a complex task, inherent to the number of subsystems involved and having to be integrated together. The available time was used to setup and tune the system, to identify problems and find solutions, and most importantly to collect knowledge on the detector behavior and how to operate it. The commissioning was completed with success and in time for the first beam. The acquired experience will prove very useful to operate the system in the future, and will help in enhancing and simplifying some procedures.

\section{References}

[1] ALICE Collaboration, Technical Proposal, CERN-LHCC-1995-71.

[2] ALICE Collaboration, The ALICE experiment at the CERN LHC, 2008 JINST 3508002

[3] ALICE Collaboration, ALICE: Physics Performance Report Vol I, J. of Phys. G : Nucl. Part. Phys 30, 1517-1763 (2004)

[4] ALICE Collaboration, ALICE: Physics Performance Report Vol II, J. of Phys. G : Nucl. Part. Phys 32, 1295-2040 (2006)

[5] J. Schukraft et al., ALICE Status and Potential, 2008 Physics at LHC - Split

[6] ALICE Collaboration, The Technical Design Report of the Trigger, Data-Acquisition, High Level Trigger, and Control System, CERN-LHCC-2003-062, January 2004. 